\title{
3D path planning based on nonlinear geodesic equation
}

\author{
Kun-Lin $\mathrm{Wu}^{1}$ Chi-Wen Lo ${ }^{1}$ Yueh-chen Lin ${ }^{1}$ and Jing-Sin Liu ${ }^{1 \dagger}$
}

\begin{abstract}
A lot of methods have been proposed for 2D path planning of mobile robot, which could be a mobile platform or a wheelchair, in planar maps. This paper addresses a concept of the shortest path planning for a mobile robot to traverse a 3D surface, which is a parametrized regular surface that models the non-flat terrain on which the mobile robot traverses. Geodesic curve linking a given start to a given target that is locally shortest on non-flat terrain is used as path. Nonlinear geodesic equations are computed by a gradient descent method with energy function of geodesic, which is shown to converge to the geodesic path in a neighborhood of target position in which a certain Lipschitz condition holds. We present numerical simulations to illustrate the geodesic path planning on non-flat terrains.
\end{abstract}

\section{INTRODUCTION}

The path planning problem is generally stated as: given a start and a goal and a description or representation of an environment, plan a path linking the start and target locations subject to some criteria of safety, mobility and optimality. The path planning for robots is a complex problem in robotics that has been studied for decades, and remain challenging in real-time robot motion in dynamic environment consisting of static or moving obstacles,such as UAV [8]-[11] and underwater robots $[11,12]$. Researchers and engineers have been interested in two-dimensional path planning for mobile robot. Many methods have been proposed for path planning, such as graph search, randomization methods, potential fields, soft computing (e.g., fuzzy logic, neural networks, evolutionary computations) based methods.

Depending on whether the environment model is completely known a priori or not, path planning is mainly classified into two categories: The first is called path planning based on the environment model or global path planning as the mobile robot knows all the information about the environment. The path could be planned offline without considering the resources of planning. The second is called path planning based on sensors or local path planning where the information of environment is provided by sensors of the robot or the environment.The robot is required to real-time plan or replan a path to account for the new information of environment gathered by the sensors and planning resources such as computing power and allowed planning time. For the first kind of path planning, we mention harmonic function [10], artifical potential field

\footnotetext{
$\dagger$ Corresponding author

1 Kun-Lin Wu, Chi-Wen Lo, Yueh-chen Lin and Jing-Sin Liu are with Institute of Information Science, Academia Sinica, Taipei, Taiwan liu@iis.sinica.edu. tw
}

[7] and heat equation etc. For the second kind of path planning, we mention wall-following [15], neural network, genetic, fuzzy and Q-learning [16] etc. The A* algorithm is between first and second [1,18]. Of all the path planning approaches for mobile robot, the assumption of a flat ground plane is assumed. The shorteset path planning on a two-dimentional plane is appealing in applications, since the path length is closely related to energy consumption and mission completion time. Among them, Dubins shortest path result on curvature constrained vehicle on 2D plane, called R-geodesic with $\mathrm{R}$ the minimum turning radius of a nonholonomic vehicle, opens a new research avenue of shortest path planning in the plane [33].

In this study, the first kind of path planning for mobile robot is considered, and we consider an issue of mobile robot motion constrained on a non-flat terrain of three-dimentional environment, such as hill climbing on a volcano or non-flat terrain in which a complete $3 \mathrm{D}$ view of the environment is adopted for mobile robot navigation. From geometric view point, the non-flat terrain in three-dimentional environment $\mathbb{R}^{3}$ that can be regarded as surface (or called regular surface) in $\mathbb{R}^{3}$ [2]. We address a concept of geodesic path used as the path primitive for locally shortest path planning on a terrain surface in $\mathbb{R}^{3}$ Euclidean space between starting point and target destination, the same as the two-dimensional environment. It is, however,not guaranteed global target convergence on terrain surface since a geodesic is defined to be locally the shortest distance from start to a target in a neighborhood of start.

From differential geometry province, we know the shortest distance between two point on a plane is the length of the section on a straight line. A geodesic can also be defined as a curve with zero geodesic curvature [19], [20]. Geodesic curvature of a curve on a surface at a point, is equal to the curvature of the normal projection of the curve onto the tangent plane of the surface at a point. A unique geodesic curve can be drawn through any point on a surface along a specified direction tangent to the surface. It can be shown that the geodesic curve segment joining start point and goal point on a surface has extremal length $[2,3,4,20]$. In the recent years, some geodesic development has Kasap [5] who used different method to solve the non-linear system of equation with boundary condition, Chen [25] proposes geodesic-like for find geodesic on surface and Gabriel [14] used geodesic distance for Riemannian metrics in computer vision and graphics. 
Before implementing simulative result, we will discretization non-linear geodesic equation system, and integrated with robotic control system such that spanning the path geodesic from start point and goal point on terrain surface. Next step, given the energy of path geodesic with gradient descent method, that is sovled numerical computation of nonlinear geodesics equation for robot dynamis. A natural option for the search direction is the nagative gradient of the energy function of geodesic path with gradient descent method, it also satified Lipschitz condition and target convengence.

The remainder of the paper is organised as follows. Section 2 introduces the geodesic approach to find shortest path for robot motion in three-dimensional environment terrain with smooth. Section 3 describes numerial computation method and target position convergence analysis. The simulations results of the geodesic path approach on several different convex terrain surface from start to target are presented in section 4 . The conclusion is given in the last section.

\section{THE GEODESIC METHODS FOR SEARCHING SHORTEST PATH}

In the Euclidean three-dimensional environment $\mathbb{R}^{3}$, there has some properties same as two-dimensional environment; namely, the robot is a point, and its position is denoted by $q_{r}=(x, y)$. The distance between position $q_{r}$ and goal $q_{g}$ is

$$
d\left(q_{r}, q_{g}\right)=\left\|q_{r}-q_{g}\right\|
$$

, where $\|\cdot\|=\langle\cdot, \cdot\rangle^{\frac{1}{2}}$ is the classical Euclidean length [2,7]. Consider the terrain surface $S$ with a parametric map $X$ : $U \rightarrow S$ of an open set $U \subset \mathbb{R}^{2}$ and two parameterized curve $C_{1}$ and $C_{2}$ on $S$, we denote $C_{1}\left(q_{s}\right): q_{s} \in I \rightarrow U$ and $C_{2}\left(q_{g}\right): q_{g} \in I \rightarrow U$, here $C_{1} \cap C_{2}=\emptyset$, where $I \in \mathbb{R}^{1}$. In order to comoputing the shortest path between start $q_{s}$ and goal $q_{g}$ on a terrain surface $S$, we establish

$$
d\left(q_{s}, q_{g}\right)=\min _{q_{s}, q_{g} \in S}\left\|X\left(C_{1}\left(q_{s}\right)\right)-X\left(C_{2}\left(q_{g}\right)\right)\right\|
$$

Thus, let $\alpha:\left[q_{s}, q_{g}\right] \subset I \rightarrow U$ be a smooth map, where $I \subset \mathbb{R}^{1}$, we obtain a smooth path curve that is shortest path $\gamma=X \circ \alpha: I \rightarrow S$, we call that is geodesic. Formally, the definition of geodesic is as below [2,4,20].

Definition 2.1: A shortest path $\gamma: I \rightarrow S$ is a parameterized geodesic path curve at $t_{0} \in I$ if $\frac{D}{d t}\left(\frac{d \gamma}{d t}\right)=0$ at the point $t_{0}$. If $\gamma$ is a geodesic at $t, \forall t \in I$, we say that $\gamma$ is a geodesic. If $\left[q_{s}, q_{g}\right] \subseteq I$ and $\gamma: I \rightarrow S$ is a geodesic, the restriction of $\gamma$ to $\left[q_{s}, q_{g}\right]$ is called a geodesic segment joining $\gamma\left(q_{s}\right)$ to $\gamma\left(q_{g}\right)$.

Where $\frac{D}{d t}$ is the covariant derivative and $\frac{d \gamma}{d t}$ is the derivative of $\gamma$ with respect to the parameter $t$. If $\gamma: I \rightarrow S$ is a geodesic, the condition $\frac{D}{d t}\left(\frac{d \gamma}{d t}\right)=0$ in the above definition means that the length of the tangent vector $\frac{d \gamma}{d t}$ is constant; i.e., the notion of a geodesic implies a curve with constant velocity and zero acceleration [2,4,20].

In addition, a terrain surface $S$ is constructed by local coordinate system, i.e., $X:(u, v) \rightarrow X(u, v) \in S$, where $X(u, v)$ be a parametrization of $S$. Therefore, the computation of the length of $\gamma$ in ambient $S \subset \mathbb{R}^{3}$, we use the first fundamental form from differential geometry, it is merely the expression of how the terrain surface $S$ inherits the natural inner product of $\mathbb{R}^{3}$. Geometrically, the first fundamental form allows us to make measurements on the surface without referring back to the ambient space $\mathbb{R}^{3}$ where the surface lies. We show it as below [2,14,20]

Definition 2.2: For a terrain surface $S$, one defines

$$
I_{S}=\langle d X, d X\rangle
$$

where $d X=X_{u} d u+X_{v} d v$. The local metric $I_{S}$ defines at each point the infinitesimal length of a path curve

$$
l(\gamma)=\int_{q_{s}}^{q_{g}}\left\|\frac{d \gamma}{d t}\right\| d t=\int_{q_{s}}^{q_{g}} \sqrt{E d u^{2}+2 F d u d v+G d v^{2}} d t
$$

where $E=\left\langle X_{u}, X_{u}\right\rangle, F=\left\langle X_{u}, X_{v}\right\rangle, G=\left\langle X_{v}, X_{v}\right\rangle$. The first fundamental form for $S$ is

$$
d s^{2}=E d u^{2}+2 F d u d v+G d v^{2}
$$

In this case, $d s$ is said to be an element of arc length.

To introduce the system of geodesic equations, that is, by $X:(u, v) \rightarrow X(u, v) \in S$, we given $\gamma(s)=$ $X(u(s), v(s)) \in S$, where the coordinate system $(u, v)=$ $(u(s), v(s))$ is orthogonal, $s \in\left[q_{s}, q_{g}\right] \subset I$, i.e., $F(u, v)=0$, which be expression of $\gamma:\left[q_{s}, q_{g}\right] \subseteq I \rightarrow S$ in the parameterization $X$ with coordinates $u(s)$ and $v(s)$. Then, the tangent vector field $\frac{d \gamma(s)}{d s}, s \in\left[q_{s}, q_{g}\right] \subset I$, is given by

$$
\frac{d \gamma(s)}{d s}=\frac{d(X(u(s), y(s))}{d s}=d u(s) X u+d v(s) X v
$$

therefore, the fact $\gamma^{\prime}(s)$ is parallel equivalent to $\frac{D}{d t}\left(\frac{d \gamma}{d t}\right)=0$

$$
\begin{array}{r}
\frac{d}{d s}\left(\frac{d \gamma(s)}{d s}\right)=u^{\prime}\left(X_{u u} u^{\prime}+X_{u v} v^{\prime}\right)+v^{\prime}\left(X_{v u} u^{\prime}+X_{v v} v^{\prime}\right) \\
+u^{\prime \prime} X_{u}+v^{\prime \prime} X_{v}
\end{array}
$$

since the covariant derivative $\frac{D}{d s}\left(\frac{d \gamma}{d s}\right)$ is the component of $\frac{d}{d s}\left(\frac{d \gamma(s)}{d s}\right)$ in the tangent plane [2,17,19], and by using Gauss Weingarten Equation for $X_{u u}, X_{u v}, X_{v u}, X_{v v}$ in terms of Christoffel symbols $\Gamma_{j k}^{i}(i, j, k=1,2)[2,5,17,20]$ are given by

$$
\begin{aligned}
& X_{u u}=\Gamma_{11}^{1} X u+\Gamma_{11}^{2} X_{v}, X_{u v}=\Gamma_{12}^{1} X u+\Gamma_{12}^{2} X_{v} \\
& X_{v u}=\Gamma_{21}^{1} X u+\Gamma_{21}^{2} X_{v}, X_{v v}=\Gamma_{22}^{1} X u+\Gamma_{22}^{2} X_{v}
\end{aligned}
$$

the fact, $\gamma^{\prime}(s)$ is parallel equivalent to the system of differential equations

$$
\begin{aligned}
& u^{\prime \prime}+\Gamma_{11}^{1}\left(u^{\prime}\right)^{2}+2 \Gamma_{12}^{1} u^{\prime} v^{\prime}+\Gamma_{22}^{1}\left(v^{\prime}\right)^{2}=0 \\
& v^{\prime \prime}+\Gamma_{11}^{2}\left(u^{\prime}\right)^{2}+2 \Gamma_{12}^{2} u^{\prime} v^{\prime}+\Gamma_{22}^{2}\left(v^{\prime}\right)^{2}=0
\end{aligned}
$$


where

$$
\begin{aligned}
\Gamma_{11}^{1} & =\frac{G E_{u}-2 F F_{u}+F E_{v}}{2\left(E G-F^{2}\right)}, \Gamma_{12}^{1}=\frac{G E_{v}-F G_{u}}{2\left(E G-F^{2}\right)} \\
\Gamma_{22}^{1} & =\frac{2 G F_{v}-G G_{u}+F G_{v}}{2\left(E G-F^{2}\right)} \\
\Gamma_{11}^{2} & =\frac{2 E E_{u}-E E_{v}+F E_{u}}{2\left(E G-F^{2}\right)} \\
\Gamma_{12}^{2} & =\frac{E G_{u}-F E_{v}}{2\left(E G-F^{2}\right)}, \Gamma_{22}^{2}=\frac{E G_{v}-2 F F_{v}+F G_{u}}{2\left(E G-F^{2}\right)}
\end{aligned}
$$

By (9) and (10) that the system is known as the differential equations of geodesics of $S$, and we have proposition as following

Proposition 2.3: Let $\alpha:\left[q_{s}, q_{g}\right] \subset I \rightarrow U$ be a smooth map of open set of $\mathbb{R}^{2}$, given the path $\gamma(s)=X \circ \alpha(s)$ : $I \rightarrow X\left(u_{1}(s), u_{2}(s)\right) \in S$ (terrain surface) is a geodesic if and only if $\gamma$ satisfies the following differential equations

$$
\frac{d^{2} u_{i}(s)}{d s^{2}}+\sum_{j, k=1}^{2} \Gamma_{i j}^{k}\left(u_{1}(s), u_{2}(s)\right) \frac{d u_{j}(s)}{d s} \frac{d u_{k}(s)}{d s}=0
$$

where $i=1,2$ and $s \in\left[q_{s}, q_{g}\right]$, both $u_{1}(s)=u(s)$ and $u_{2}(s)=v(s)$ are local coordinate system of $S$.

Now we show that a geodesic minimizes arc length of path for points sufficiently close. In addition, if a path minimizes arc length between $q_{s}$ and $g_{g}$, it is a geodesic $[2,4,20]$

Definition 2.4: A segment of the geodesic path $\gamma:\left[q_{s}, q_{g}\right] \rightarrow S$ is called minimizing if $l(\gamma) \leq l(\mu)$, where $l(\cdot)$ denotes the length of path and $\mu$ is an arbitrary piecewise differentiable path joining $\gamma\left(q_{s}\right)$ to $\gamma\left(q_{g}\right)$.

By Def. 2.4, the geodesics locally minimize the arc length that is given $[2,4,20]$

Proposition 2.5: Let $p \in S, U$ be a neighborhood of $p$, and $B \subset N$ a ball of center $p$. Let $\gamma:\left[q_{s}, q_{g}\right] \rightarrow B$ be a geodesic segment with $\gamma\left(q_{s}\right)=p$. If $\mu:\left[q_{s}, q_{g}\right] \rightarrow S$ is any piecewise differentiable path joining $\gamma\left(q_{s}\right)$ to $\gamma\left(q_{g}\right)$ then $l(\gamma) \leq l(\mu)$ and if equality holds then $\gamma\left(\left[q_{s}, q_{g}\right]\right)=\mu\left(\left[q_{s}, q_{g}\right]\right)$

From above, the shortest path distance between two objects on a terrain surface $S$ is defined by the lengthminimizing path from the start $q_{s}$ to the goal $q_{g}$ on $S$. We obtain

$$
d\left(q_{s}, q_{g}\right)=\inf _{\gamma \in \Omega} l(\gamma)
$$

where $\Omega$ is the set of all paths from the start $q_{s}$ to the goal $q_{g}$ on a terrain surface $S$, and the set $\Omega$ can be only considered the set of all geodesic on $S$ from $q_{s}$ to $q_{g}$.

\section{COMPUTATION OF ROBOTIC GEODESICS AND CONVERGENCE ANALYSIS}

Let the robotic state $q_{r} \in \mathbb{R}^{n}$ and the velocity (control law) $\nu^{r} \in \mathbb{R}^{n}$ [22]. In addition, from Prop. 2.3 the two second-order differential equations can be rewritten as four first-order differential equations [19]

$$
\frac{d u_{i}(s)}{d s}=\nu_{i}^{r}, i=1,2
$$

and derivative of $\nu_{i}^{r}$, we have

$$
\frac{d \nu_{i}^{r}}{d s}=-\sum_{j, k=1}^{2} \Gamma_{i j}^{k}\left(u_{1}(s), u_{2}(s)\right) \frac{d u_{j}(s)}{d s} \frac{d u_{k}(s)}{d s}, i=1,2
$$

that we can obtain control system for robot

$$
\dot{q}_{r}(s)=\nu_{i}^{r}, \quad i=1,2
$$

and

$\dot{\nu}_{i}^{r}=\sigma=-\sum_{j, k=1}^{2} \Gamma_{i j}^{k}\left(u_{1}(s), u_{2}(s)\right) \frac{d u_{j}(s)}{d s} \frac{d u_{k}(s)}{d s}, i=1,2$

where $\sigma$ is the control input [23].

By (17), the robotic route is spanning path from two points, start and goal, on the terrain surface $S$, that is solution of geodesic equations, or which path is geodesic on the terrain surface. Now we're going to discretize the the geodesic equations (9) and (10) governing the geodesic path linking start and goal with the following bouondary conditions after discretization [5]

$$
u\left(q_{s}\right)=u_{0}, v\left(q_{s}\right)=v_{0}, u\left(q_{g}\right)=u_{n+1}, v\left(q_{g}\right)=v_{n+1}
$$

where $(u, v) \in U \subset \mathbb{R}^{2}$. If $\left\{u_{i}, v_{i}\right\}_{i=0}^{n+1}$ is a solution of system of geodesic differential equations (9) and (10) with $X\left(u_{0}, v_{0}\right)=q_{0}=q_{s}$ and $X\left(u_{n+1}, v_{n+1}\right)=q_{n+1}=q_{g}$, then the $\left\{X\left(u_{i}, v_{i}\right)\right\}_{i=0}^{n+1}$ would approach the geodesic $\gamma$ as $n+1$ approaches to infinity [6,24]. Therefore, the set $\left\{q_{s}=\right.$ $\left.q_{0}, q_{1}, \cdots, q_{n}, q_{n+1}=q_{g}\right\}$ can be represented as the state of robotic position with respect to arc-length $s$ (we assume the arc-length $s$ is proportional to time T, i.e. $s(T)=T(s)$ ) on the terrain surface $S$. Using the concept of Potential Field Methods which the robot can described as

$$
\nabla q_{r}=\left\|q_{r}-q_{n}\right\|=0, \forall n \in \mathbb{N}
$$

where the robotic position currently can regarded as the new start point $q_{s}$ for robot.

From preceding section, a terrain surface $S$ is represented as a parametric surface with parametrization $X: U \rightarrow S$, and consider $\gamma:\left[q_{s}, q_{g}\right] \rightarrow U$ be a path geodesic on $S$, the energy of geodesic path $\gamma$ we have $[25,30]$

$$
E(\gamma)=\frac{1}{2} \int_{q_{s}}^{q_{g}}\left\|\frac{d \gamma}{d s}\right\|^{2} d s=\frac{1}{2} \int_{q_{s}}^{q_{g}}\left\langle X^{\prime}, X^{\prime}\right\rangle d s
$$


With $\left\{u_{i}, v_{i}\right\}_{i=0}^{n+1}$, is a solution of system of geodesic differential equations, the engergy function $E$ with respect to the parameter $s[25,26,30]$, we obtain

$$
E\left(u_{i}, v_{i}\right)=\frac{1}{2} \int_{q_{s}}^{q_{g}}\left\langle X^{\prime}(u(s), v(s)), X^{\prime}(u(s), v(s))\right\rangle d s
$$

differentiating $E$ with respect to $u_{i}$ and $v_{i}$ respectively, we obtain [25]

$$
\begin{aligned}
& E_{u_{i}}=\int_{q_{s}}^{q_{g}}\left\langle\frac{\partial}{\partial u_{i}} X^{\prime}(u(s), v(s)), X^{\prime}(u(s), v(s))\right\rangle d s \\
& E_{v_{i}}=\int_{q_{s}}^{q_{g}}\left\langle\frac{\partial}{\partial v_{i}} X^{\prime}(u(s), v(s)), X^{\prime}(u(s), v(s))\right\rangle d s
\end{aligned}
$$

where

$$
\begin{aligned}
& \frac{\partial}{\partial u_{i}} X^{\prime}(u(s), v(s))=\frac{\partial}{\partial u_{i}}\left(X_{u} u^{\prime}+X_{v} v^{\prime}\right) \\
& \frac{\partial}{\partial v_{i}} X^{\prime}(u(s), v(s))=\frac{\partial}{\partial v_{i}}\left(X_{u} u^{\prime}+X_{v} v^{\prime}\right)
\end{aligned}
$$

Therefore, the gradient of energy function $E$ is $[25,26,30]$

$$
\nabla E=\left(E_{u_{0}}, E_{u_{1}}, \cdots, E_{u_{n}}, E_{v_{0}}, E_{v_{1}}, \cdots, E_{v_{n}}\right)
$$

where $E_{u_{i}}$ and $E_{v_{i}}$ are the partial derivative of energy function $E$, by [25], we have $\nabla E\left(u_{i}, v_{i}\right)$ is a decreasing direction.

To compute the locally shortest path for robot motion on a non-flat terrain, we would apply the gradient descent method with the energy of geodesic path. The description for gradient descent method with the energy of geodesic path is

$$
q_{n+1}=q_{n}-\beta_{n} \nabla E\left(q_{n}\right), \forall n \in \mathbb{N}
$$

where $\beta_{n}>0$ is called step size and $\nabla E$ is the gradient of $E$ and $\nabla E\left(q_{n}\right)$ is

$\nabla E\left(q_{n}\right)=\nabla E\left(\gamma\left(u_{n}, v_{n}\right)\right)=\nabla\left(\frac{1}{2} \int_{q_{s}}^{q_{g}}\left\|\frac{\partial}{\partial s} X\left(u_{n}, v_{n}\right)\right\|^{2} d s\right)$

Obviously, the sequence $\left\{q_{n}\right\}$ is bounded. Moreover, $\left(-\nabla E\left(q_{n}\right)\right)$ is a descent direction. Now, we are going to show that $E$ will converge to a stationary point. For the convergence, we assume that the gradient of energy function $E$ satisfies a Lipschitz continuous with exists a constant $C>0$, such that

$$
\left\|\nabla E\left(q_{n+1}\right)-\nabla E\left(q_{n}\right)\right\| \leq C\left\|q_{n+1}-q_{n}\right\|, \forall q_{n+1}, q_{n}
$$

Now consider the expansion of $E$. From [27,29,31], we have

$$
\left\|E\left(q_{n+1}\right)-E\left(q_{n}\right)-\left\langle\nabla E\left(q_{n}\right), q_{n+1}-q_{n}\right\rangle\right\| \leq \frac{C}{2}\left\|q_{n+1}-q_{n}\right\|^{2}
$$

For applying the gradient descent method (26), too large $\beta_{n}$ could cause divergence. Thus, we fixed $\beta_{n} \in(0,1]$, start with $\beta_{n}=\beta=1$ and $\beta<\frac{2}{C}$ [28]. Applying (26) into (29), we obtain $[27,28,29,31]$

$E\left(q_{n+1}\right) \leq E\left(q_{n}\right)+\left\langle\nabla E\left(q_{n}\right), q_{n+1}-q_{n}\right\rangle+\frac{C}{2}\left\|q_{n+1}-q_{n}\right\|^{2}$ where $\left(q_{n+1}\right)$ is satisfied (26), it lead to

$$
E\left(q_{n+1}\right) \leq E\left(q_{n}\right)-\beta\left\|\nabla E\left(q_{n}\right)\right\|^{2}+\frac{\beta^{2} C}{2}\left\|\nabla E\left(q_{n}\right)\right\|^{2}
$$

after shift, we have

$$
\left\|\nabla E\left(q_{n}\right)\right\|^{2} \leq \frac{E\left(q_{n}\right)-E\left(q_{n+1}\right)}{\beta\left(1-\frac{\beta C}{2}\right)}
$$

sum of $\left\|\nabla E\left(q_{n}\right)\right\|^{2}$, we obtain

$$
\sum_{i=0}^{n+1}\left\|\nabla E\left(q_{n}\right)\right\|^{2} \leq \frac{E\left(q_{0}\right)-E\left(q_{n+1}\right)}{\beta\left(1-\frac{\beta C}{2}\right)}
$$

which can be rewritten as $[27,28,29,31]$

$$
\lim _{n \rightarrow \infty} \nabla E\left(q_{n}\right)=0
$$

From this, we deduce that the energy of function $E$ is convergent with the gradient descent method. On the other hand, [28] also showed that $E\left(q_{n}\right)$ is decreasing and convergent. Furthermore, by [25,26], we can obtain $\nabla E\left(u_{i}, v_{i}\right) \rightarrow$ 0 with $q_{n+1}=q_{n}$ when $n$ is large enough, that isthe robot eventually reach the goal $q_{g}$ along the geodesic path direction, and the energy is decreasing from the start to its local minimum at the target.

\section{SIMULATION}

For our simulations in this section, the map is the smoothing, free-obstacle convex terrain surface locally, as Fig. 1 and Fig. 2 depict. Three different routes from start position $(0,0,0)^{1}$,to fixed target on two different three-dimensional maps for geodesic path planning, where the sign "+" and the " $\square$ " denote start and target in the sample maps, respectively. Dotted line indicates a computed shortest path. The target is set as $(2.5,1,5)$ for Fig. 1 and $(2,2,0)$ for Fig. 2, respectively.

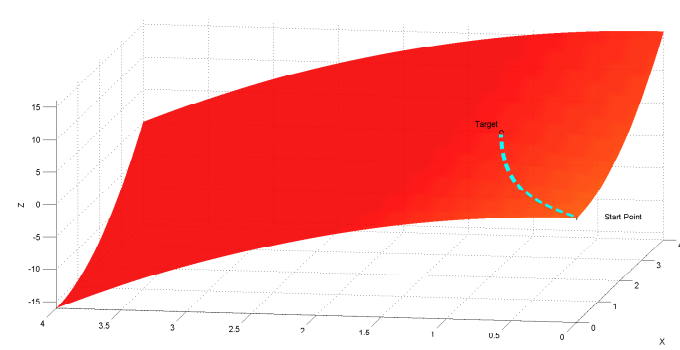

Fig. 1. The robotic route hails from initial $(0,0,0)$ to target that at upper region on the terrain suface

The shortest path Fig. 3 seems to downhill by initial $(0,0,0)$ to target $(1.5,3.5,-30)$, which is distinct from Fig. 1 and Fig. 2. Even though Fig. 1 and Fig. 2 display the routes on the same terrain, one route goes like as an uphill climbing with elevation change, while the other route goes straight without elevation change. That is exactly shortest path for robot

\footnotetext{
${ }^{1}(x, y, z)$ is represented as Euclidean coordinate space
} 


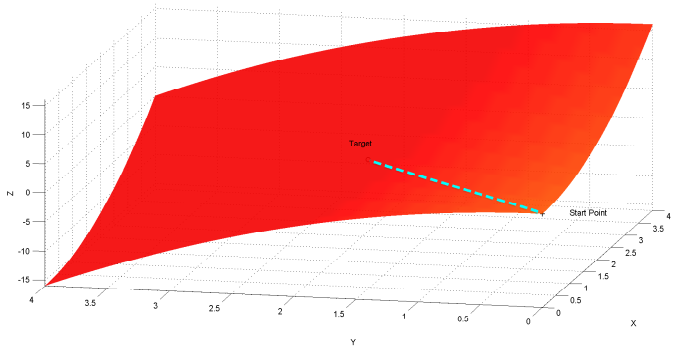

Fig. 2. The robotic route to target in alignment with respect to initial position $(0,0,0)$

moves to target, due to geodesic is a curve on parametric surface generalizing the notion of a shortest path between a given start and target in the planar environment. Note that it is only true locally, in other word, the robot path could not make sure a shortest path globally. In general setting, discrete piecewise paths joining an ordered list of sub-targets is a concise way for planning in a global environment map, e.g., chosen by human control i.e.

$$
\mathcal{T}=\sum_{i=1}^{n+1}\left\|\mathcal{T}_{i}-\mathcal{T}_{i-1}\right\|
$$

where $\mathcal{T}, \mathcal{T}_{i}$ and $n+1$ indicate target, sub-targets and a number of segment or node control respectively. Thus, the robot could move from start to target sequentially along a series of segmented connections via sub-targets using geodesic by prop. 2.5, thus guaranteeing a shortest path segment in each movement. However, coming to grips with amid the problems by power consumption effectiveness and safe navigation of mobile robot in dangerous terrain or region in practice, discrete piecewise geodesic path is a way of overcoming the problem of power consumption constraint and avoiding dangeous region in authentic situation simultaneously, while maintaining a shortest distance of each movement toward target.

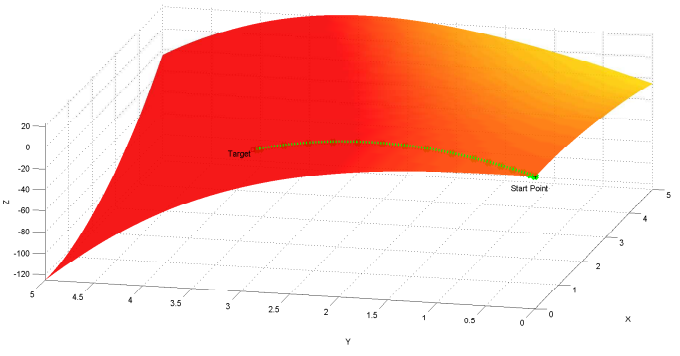

Fig. 3. The robotic route hails from initial $(0,0,0)$ to target that at lower region on the terrain surface

\section{CONCLUSIONS}

In this study, we have introduced the locally shortest path planning for mobile robot motion on a non-flat terrain using geodesic approach, and used a gradient descent method with energy function of geodesic path curve to solve numerically the nonlinear geodesic equation with boundary condition and robot dynamics. The gradient descent method with geodesic path energy function satisfying certain Lipschitz conditions achieves local target convergence along a geodesic path. Although shortest path planning for robot motion is ensuredonly locally, discrete piecewise path segements connecting an ordered list of sub-targets specified by human or other heuristics can enable perfoming global path planning in the presence of obstacles to reach a target in 3D geometric workspace. For future extensions of the work presented in this paper, we will study the power consumption effectiveness for safe path planning in more complex 3D envrionment, in addition to improvement in both numerical analysis and computation of shortest path.

\section{REFERENCES}

[1] Ting-Kai Wang, Quan Dang and Pei-Yuan Pan, Path Planning Approach in Unknown Environment, International Journal of Automation and Computing, 7(3), August 2010, 310-316.

[2] Manfredo P. do Carmo Differential Geometry of Curves and Surfaces, 1976, Prentice-Hall, Englewood Cliffs, New Jersey

[3] G.V.V. Ravi Kumar, Prabha Srinivasan, V. Devaraja Holla, K.G. Shastry, Geodesic Curve Computations on Surfaces Computer Aided Geometric Design, 20(2003), 119-133

[4] Lo Yu-Yun, A Geometric Method for Searching Geodesics on Regular Surfaces, Department of Mathematics, National Chung Cheng University, 2004

[5] Emin Kasap, Mustafa Yapici, F. Talay Akyildiz, A Numerical Study for Computation of Geodesic Curves, Applied Mathematics and Computation, 171(2005), 1206-1213

[6] Zhi-Teng Zheng, Extended Kasap's Method for Estimating the Boundary Geodesic Problem on a Regular surface, Department of Applied Mathematics, National Chiayi University, 2011

[7] S. S. Ge, Y. J. Cui, New Potential Functions for Mobile Robot Path Planning, IEEE Transactions on Robotics and Automation, Vol. 16, No. 5, October 2000

[8] Chen Friedman, Inderjit Chopra, Svetlana Potyagaylo, Omri Rand, Towards Obstacle Avoidance and Autonomous UAV Operation,Proc. of AHS International Meeting on Advanced Rotorcraft Technology and Safety Operations (HeliJapan), Phoenix, AZ. AHS International, January 25-27, 2011

[9] Omer Cetin, Ibrahim Zagli, Guray Yilmaz, Establishing Obstacle and Collision Free Communication Relay for UAVs with Artificial Potential Fields, Journal of Intelligent \& Robotic Systems, Volume 69, Issue 1-4, January 2013, pp 361-372

[10] Ahmad A. Masoud, A Harmonic Potential Field Approach for Planning Motion of a UAV in a Cluttered Environment with a Drift Field, 2011 50th IEEE Conference on Decision and Control and European Control Conference (CDC-ECC) Orlando, FL, USA, December 12-15, 2011

[11] Ahmad A. Masoud, A Harmonic Potential Approach for Simultaneous Planning and Control of a Generic UAV Platform, A Harmonic Potential Approach for Simultaneous Planning and Control of a Generic UAV Platform, Journal of Intelligent \& Robotic Systems, Volume 65 , Issue 1-4, pp 153-173, January 2012.

[12] C. Pêtrès, Y. Pailhas, P. Patrón, Y. Petillot, J. Evans, D. Lane, Path Planning for Autonomous Underwater Vehicles, IEEE Transactions on Robotics, Volume 23, Issue 2, April 2007, Page 331-341

[13] C. Pêtrès, Y. Pailhas, Y. Petillot, D. Lane, Underwater path planing using fast marching algorithms, in Proceedings of the Oceans Europe, vol. 2, pp. 814-819, June 2005.

[14] Gabriel Peyré, Laurent D. Cohen, Geodesic Methods for Shape and Surface Processing, Advances in Computational Vision and Medical Image Processing, Computational Methods in Applied Sciences Volume 13, 2009, pp 29-56

[15] I. Gavrilut, V. Tiponut, A. Gacsadi, L. Tepelea, Wall-following Method for an Autonomous Mobile Robot using Two IR Sensors, 12th WSEAS International Conference on SYSTEMS, Heraklion, Greece, July 2224, 2008 
[16] Hisao Ishibuchi, Tomoharu Nakashima, Hiromitsu Miyamoto, ChiHyon Oh, Fuzzy Q-Learning for a multi-player non-cooperative repeated game, Fuzzy Systems, 1997, Proceedings of the Sixth IEEE International Conference, page 1573-1579, vol.3

[17] Nassar H. Abdel-All, E. I. Abdel-Galil, Numerical Treatment of Geodesic Differential Equations on a Surface in $\mathbf{R}^{3}$, International Mathematical Forum, Vol. 8, 2013, no. 1, 15 - 29, 2013

[18] Dilshat Saitov, Suk Gyu Lee, Coordinated A* Algorithm In Multi Robot System, International Conference on Advances in Electrical and Electronics Engineering (ICAEE'2011), pp.165-168, 2011

[19] Nicholas M. Patrikalakis, Takashi Maekawa, Shape Interrogation for Computer Aided Design and Manufacturing, Springer-Verlag Berlin Heidelberg 2002

[20] Victor A. Toponogov, Differential Geometry of Curves and Surfaces : A Concise Guide, Birkhauser-Verlag, 2006

[21] Wongvisarut Khuangsatung, Pakkinee Chitsakul, Some Geodesics in Open Surfaces Classified by Clairaut's Relation, World Academy of Science, Engineering and Technology 692012

[22] M. A. El Kamel, L. Beji, A. Abichou, S. Mammar, A Novel Obstacle Avoidance Approach for Mobile Robot System Including Target Capturing, INTELLIGENT SYSTEMS AND AUTOMATION: 2nd Mediterranean Conference on Intelligent Systems and Automation (CISA'09). AIP Conference Proceedings, Volume 1107, pp. 249-253 (2009)

[23] Jiangmin Chunyu, Zhihua Qu, Eytan Pollak, Mark Falash, A New Reactive Target-tracking Control with Obstacle Avoidance in a Dynamic Environment, 2009 American Control Conference Hyatt Regency Riverfront, St. Louis, MO, USA June 10-12, 2009

[24] Zheng, Z. T.and Chen, S. G., The general orthogonal projection on a regular surface. In Proceedings of the 5th European conference on European computing conference (pp. 116-119), 2011. World Scientific and Engineering Academy and Society (WSEAS).

[25] Sheng-Gwo Chen, Geodesic-like curves on parametric surfaces, computer Aided Geometric Design, 27 (2010) 106-117

[26] Wen-Haw Chen, Sheng-Gwo Chen, Computation of the shortest path between two curves on a parametric surface by geodesic-like method, arXiv preprint arXiv:1011.5320.
[27] http://www.stat.purdue.edu/ vishy/introml/notes/Optimization.pdf

[28] Regina Burachik, Luis Mauricio Graña Drummond, Alfredo N. Iusem, B.F. Svaiter, Full convergence of the steepest descent method with inexact line searches, Optimization, 32(2), 137-146

[29] Stephen Boyd, Lieven Vandenberghe, Convex Optimization, Cambridge University Press, 2004

[30] Wen-Haw Chen, Sheng-Gwo Chen, A note of boundary geodesic problem on regular surfaces, Proceedings of the European Computing Conference, In Proceedings of the 5th European conference on European computing conference (pp. 105-109). World Scientific and Engineering Academy and Society (WSEAS).

[31] http://users.ece.utexas.edu/ cmcaram/EE381V_2012F/Lecture_4_Scri be Notes.final.pdf

[32] Ninad Pradhan, Timothy Burg, Stan Birchfield, Ugur Hasirci, Indoor navigation for mobile robots using predictive fields, American Control Conference (ACC), 2013, 3237-3241.

[33] Steven Michael LaValle. Planning algorithms. Cambridge university press, 2006 